\title{
Survey of fish species from plateau streams of the Miranda River Basin in the Upper Paraguay River Region, Brazil
}

\author{
Fabiane Silva Ferreira', Gabriela Serra do Vale Duarte ${ }^{1}$, Francisco Severo-Neto ${ }^{2}$, Otávio Froehlich ${ }^{3}$ \& \\ Yzel Rondon Súarez** \\ ${ }^{1}$ Universidade Estadual de Mato Grosso do Sul, Centro de Estudos em Recursos Naturais, Dourados, MS, Brazil \\ ${ }^{2}$ Universidade Federal de Mato Grosso do Sul, Laboratório de Zoologia, Campo Grande, CG, Brazil \\ ${ }^{3}$ Universidade Federal de Mato Grosso do Sul, Departamento de Zoologia, Campo Grande, CG, Brazil \\ ${ }^{4}$ Universidade Estadual de Mato Grosso do Sul, Centro de Estudos em Recursos Naturais, Lab. Ecologia, \\ Dourados, MS, Brazil \\ *Corresponding author: Yzel Rondon Súarez,e-mail: yzel@uems.br
}

FERREIRA, F. S., DUARTE, G. S. V., SEVERO-NETO, F., FROEHLICH O., SÚAREZ, Y. R. Survey of fish species from plateau streams of the Miranda River Basin in the Upper Paraguay River Region, Brazil. Biota Neotropica. 17(3): e20170344. http://dx.doi.org/10.1590/1676-0611-BN-2017-0344

\begin{abstract}
The objective of this study was to provide a comprehensive list of the fish fauna of headwater streams of the Miranda River in the Upper Paraguay River Basin. Our primary data set was constructed from sampling of fish using a rectangular sieve, trawl, and gill nets from 2004 to 2015. Our secondary data were derived from published reports conducted in the Miranda River Basin, in addition to taxonomic and distribution data from other studies conducted in the basin. All data were compiled, which in the end encompassed a period from 1999 to 2015 . The datasets yielded a total of 143 species, 104 from the primary data (72.7\%) and 39 from the secondary data (27.3\%). Species were distributed among seven orders and 30 families were found in the Miranda River Basin. Characiformes and Siluriformes were the predominant orders, and the families Characidae and Loricariidae had the greatest number of species. Our results indicate a greater number of species compared to other studies of the Upper Paraguay Basin headwaters, likely due to the longer time frame covered by our primary and secondary datasets.
\end{abstract}

Keywords: Species List, Pantanal, streams ichthyofauna, plateau, Paraguay basin.

\section{Levantamento das espécies de peixes da bacia do Rio Miranda em riachos do planalto, Alto Rio Paraguai, Brasil}

\begin{abstract}
Resumo: O objetivo deste trabalho é o de prover uma listagem da ictiofauna de riachos de cabeceira da bacia do Rio Miranda, Alto Rio Paraguai. Os peixes foram amostrados utilizando uma peneira retangular, rede de arrasto e rede de espera no período de 2004 a 2015, além disso, outros trabalhos realizados na bacia do Rio Miranda foram compilados e adicionados na listagem total, assim como, artigos de descrição e distribuição de espécies amostradas na bacia compreendendo o período de 1999 a 2015. A bacia do Rio Miranda apresentou um total de 143 espécies, 104 espécies oriundas de dados primários $(72,7 \%)$ e 39 de dados secundários (27,3\%). As espécies estão distribuídas em sete ordens e 30 famílias. Characiformes e Siluriformes foram as ordens predominantes e as famílias Characidae e Loricariidae apresentaram maior número de espécies. Os resultados obtidos neste estudo demonstram um maior número de espécies quando comparados com outros estudos realizados em cabeceiras na bacia do Alto Rio Paraguai decorrente ao longo período de estudo.
\end{abstract}

Palavras-chave: Lista de espécies, Pantanal, ictiofauna de riachos, planalto, bacia do Paraguai.

\section{Introduction}

The Paraguay River Basin is an important drainage of the La Plata River Basin (Lowe-McConnell 1999), and occupies a total area of about $496,000 \mathrm{~km}^{2}$ (Junk et al. 2006). The Brazilian Pantanal has a total area of approximately $361,700 \mathrm{~km}^{2}$, with $72.7 \%$ of the total territory lying in Brazil and the remainder in Bolivia and Paraguay. The upper Paraguay basin in Brazilian divided into two distinct regions: a floodplain known as the 'Pantanal', which at approximately 138,200 $\mathrm{km}^{2}$ constitutes one of the largest wetland areas in the world; and a plateau comprised of areas with altitudes greater than $200 \mathrm{~m}$ encompassing approximately $223,500 \mathrm{~km}^{2}$ (Silva \& Abdon 1998). These two regions are distinguished by geological and geomorphological characteristics, however they are also interdependent since the headwaters of the most important rivers 
draining into the Pantanal are in the plateau region, resulting in strong hydrologic connectivity (Willink et al. 2000, Teresa et al. 2010).

Approximately 270 fish species have been identified in the Paraguay River Basin (Britski et al. 2007). However, the total number of species would likely be higher if the plateau streams had been included (Alho \& Sabino 2012). Numerous studies have been carried out in the basin over the past two decades, including the floodplain region (Catella \& Petrere-Jr 1996, Willink et al. 2000, Junk et al. 2006, Baginski et al. 2007, Pacheco \& Silva 2009, PainsSilva et al. 2010, Tondato et al. 2013, Súarez et al. 2013, Pains-Silva et al. 2014, Polaz et al. 2014, Botini et al. 2015 and Severo-Neto et al. 2015) and plateau waters (Súarez et al. 2007, Terra \& Sabino 2007, Teresa et al. 2010, Casatti et al. 2010, Teresa \& Romero 2010, Castro \& Vizzotto 2013, Krinski et al. 2015 and Oliveira et al. 2015). In studies of the plateau portion of the basin, analyses at small spatial scales were the predominant.

Compared to Brazilian hydrographic basins (e.g. the Upper Paraná River system), the fish fauna of central Brazil is poorly described (Casatti et al. 2010). Despite being a hotspot for endemic fauna (Willink et al. 2000) the main Pantanal headwater streams are understudied. The goal of this study was thus to describe the ichthyofauna of plateau region streams of the Miranda River Basin by combining primary and secondary data compiled from previous studies on the basin.

\section{Materials and Methods}

\section{Study site}

The Miranda River Basin is entirely within the limits of the Brazilian region, in Mato Grosso do Sul State. The total basin area is $42,993.83 \mathrm{~km}^{2}$, with Serra da Bodoquena as the western boundary and the Paraguay River as the northwestern boundary (Plano de Recursos Hídricos da Bacia Hidrográfica do Rio Miranda 2014). The main tributaries are the Miranda and Aquidauana Rivers. The rivers and streams of the Aquidauana and Miranda Rivers flow into the Brazilian Cerrado (savanna), which is composed of patches of deciduous, semi-deciduous, and riparian forest that vary in density depending on the ecological drainage system in which they lie (Jesus 2003, Junk et al. 2006).

Surveys were conducted at 42 sites in the Miranda River Basin from January 2004 to January 2015 (primary data). Additional secondary data were compiled from 16 collection sites from published work on stream fish assemblages in the Miranda River Basin (Willink et al. 2000, Casatti et al. 2010, Teresa et al. 2010, Teresa \& Romero 2010). These 58 sampling sites are distributed among streams from first to third order in 18 municipalities in Mato Grosso do Sul, Brazil (Figure 1). Taxonomic and distribution data from additional studies were also added to the final species list (Sabino \& Trajano 1997, Willink et al. 2000, Ribeiro et al. 2007, Terra \& Sabino 2007, Zawadzki et al. 2014, Shibatta 2016).

\section{Sampling}

For the primary data set, fish were sampled predominantly using a $0.8 \times 1.2 \mathrm{~m}$ rectangular sieve and $5 \times 1.5 \mathrm{~m}$ trawl nets, both with $2 \mathrm{~mm}$ mesh. In streams with a higher volume, sampling was supplemented with $10 \times 1.5 \mathrm{~m}$ gill nets with mesh sizes of 15, 25, 30, 40, and $50 \mathrm{~mm}$ between adjacent knots. Sampled fish were anesthetized with Eugenol solution and subsequently fixed by immersion in 4\% formaldehyde solution for at least 48 hours. Specimens were then washed and transferred to $70 \%$ ethanol.

Fish identification was performed mainly using the Pantanal fish identification key of Britski et al. (2007), and specific keys were used when necessary. Voucher specimens were deposited in the fish collection at the Universidade Federal de Mato Grosso do Sul (ZUFMS/UFMS) and the Núcleo de Pesquisa em Limnologia, Ictiologia e Aquicultura at the Universidade Estadual de Maringá (Nupelia/UEM). For the secondary data set, obtained by published articles and fish collection, voucher specimens were deposited in the fish collection of the Departamento de Zoologia e Botânica at the Universidade Estadual Paulista, São José do Rio Preto (DZSJRP/UNESP), Fish collection of Laboratório de Ictiologia de Ribeirão Preto (LIRP/USP) and Fish collection of Museu de Zoologia of University of Londrina (MZUEL/UEL). Collections were authorized by IBAMA (collection permit number SISBIO; process number 13458-1). Fish classification follows Eschmeyer et al. (2017).

\section{Data analysis}

To evaluate sampling efficiency, the total richness in the basin was estimated using a species accumulation curve with the bootstrap method (Smith \& Van Belle 1984), as well as standard error using the function 'specaccum' in the 'vegan' package (Oksanen et al. 2011) in R (R Development Core Team 2013).

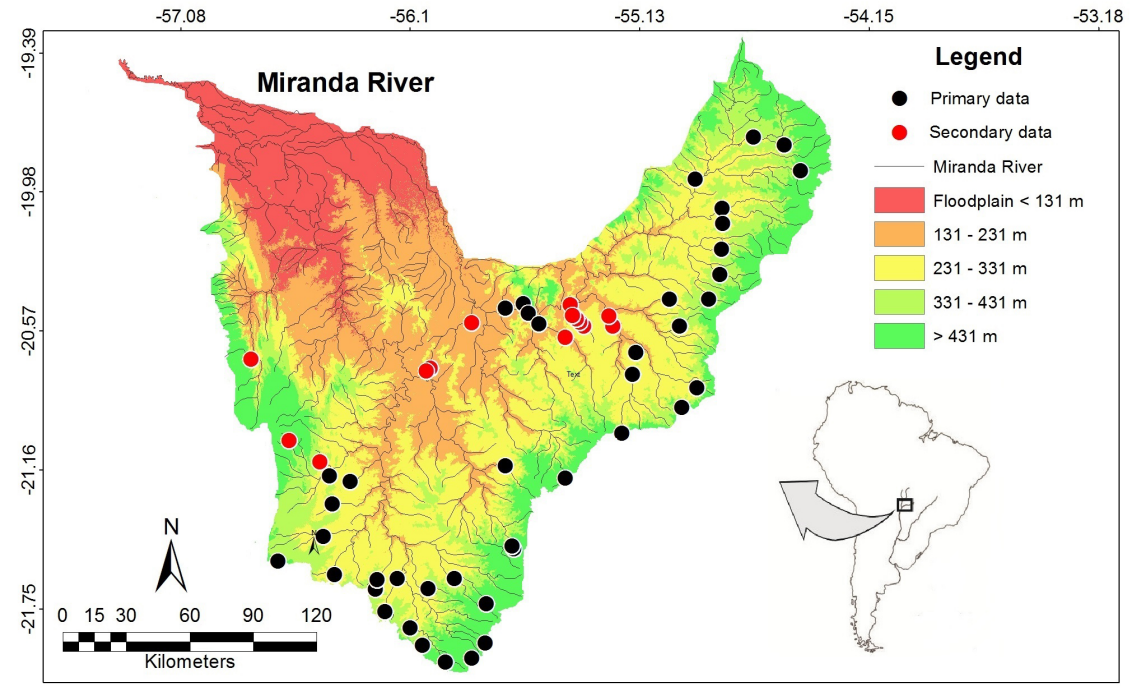

Figure 1: Locations of the sampling points in the Miranda River Basin of the Upper Paraguay River, Mato Grosso do Sul, Brazil. Black dots: Primary data; Red dots: Secondary data. 


\section{Results}

The datasets yielded a total of 143 species, 104 from the primary data $(72.7 \%)$ and 39 from the secondary data $(27.3 \%)$ data. Species were distributed among seven orders and 30 families (Table 1). Characiformes ( 75 species) and Siluriformes (48 species) were the predominant orders, representing together $86 \%$ of the recorded species. Among the families of these orders, the largest numbers of species were from Characidae and Loricariidae, representing 32.2\% (46 species) and $15.4 \%$ (22 species) of the total sample, respectively (Figure 2).
The species accumulation curve shows that richness does not reach the asymptotic threshold (Figure 3), indicating the need for further collection efforts (i.e., increasing the number of sampling sites should increase estimates of species richness). The bootstrap method estimated approximately 169 with confidence interval $(\alpha=0.05)$ from 150 to 188 species, represent approximately $84.6 \%$ of the estimated by datasets (primary and secondary) the total number of species in the Miranda River basin.

In relation the species sampled Piaractus mesopotamicus (Holmberg 1887), Pseudoplatystoma corruscans (Linnaeus 1766) are on the National List

Table 1: Final list of species from sampled and compiled data from the Miranda River Basin, Upper Paraguay River. Location of voucher deposition: DZSJRP (Departamento de Zoologia e Botânica da Universidade Estadual Paulista, São José do Rio Preto - UNESP); ZUFMS (Coleção Zoológica da Universidade Federal do Mato Grosso do Sul - UFMS); LIRP (Coleção de Peixes do Laboratório de Ictiologia de Ribeirão Preto, Universidade de São Paulo - USP); MZUEL (Museu de Zoologia da Universidade Estadual de Londrina - UEL); NUP (Núcleo de Pesquisa em Limnologia, Ictiologia e Aquicultura da Universidade Estadual de Maringá - Nupelia/UEM).

\begin{tabular}{|c|c|}
\hline Taxon & Voucher \\
\hline \multicolumn{2}{|l|}{ BELONIFORMES } \\
\hline \multicolumn{2}{|l|}{ Belonidae } \\
\hline Potamorrhaphis eigenmanni Miranda-Ribeiro 1915 & DZSJRP-12880 \\
\hline \multicolumn{2}{|l|}{ CHARACIFORMES } \\
\hline \multicolumn{2}{|l|}{ Acestrorhynchidae } \\
\hline Acestrorhynchus pantaneiro Menezes 1992 & ZUFMS-PIS01657 \\
\hline \multicolumn{2}{|l|}{ Anostomidae } \\
\hline Leporinus friderici (Bloch 1794) & ZUFMS-PIS01063 \\
\hline Leporinus lacustris Campos 1945 & DZSJRP-12439 \\
\hline Leporinus striatus Kner 1858 & DZSJRP-12438 \\
\hline Leporellus vittatus (Valenciennes 1850) & ${ }^{5}$ LIRP-5334 \\
\hline Megaleporinus obtusidens (Valenciennes 1836) & DZSJRP-12858 \\
\hline \multicolumn{2}{|l|}{ Bryconidae } \\
\hline Brycon hilarii (Valenciennes 1849) & DZSJRP-11992 \\
\hline Salminus brasiliensis (Cuvier 1816) & DZSJRP-000534 \\
\hline \multicolumn{2}{|l|}{ Characidae } \\
\hline Galeocharax humeralis (Valenciennes 1834) & ZUFMS-PIS03418 \\
\hline Roeboides descalvadensis Fowler 1932 & DZSJRP-12871 \\
\hline Phenacogaster tegatus (Eigenmann 1911) & ZUFMS-PIS03802 \\
\hline Odontostilbe pequira (Steindachner 1882) & ZUFMS-PIS03908 \\
\hline Odontostilbe paraguayensis Eigenmann \& Kennedy 1903 & ZUFMS-PIS03815 \\
\hline Serrapinnus calliurus (Boulenger 1900) & ZUFMS-PIS03847 \\
\hline Serrapinnus kriegi (Schindler 1937) & ZUFMS-PIS01399 \\
\hline Serrapinnus microdon (Eigenmann 1915) & ${ }^{5}$ MZUEL-12591 \\
\hline Serrapinnus notomelas (Eigenmann 1915) & ZUFMS-PIS04040 \\
\hline \multicolumn{2}{|l|}{ Characidae Incertae Sedis } \\
\hline Aphyocharax anisitsi Eigenmann \&Kennedy 1903 & ZUFMS-PIS01275 \\
\hline Aphyocharax dentatus Eigenmann \& Kennedy 1903 & ZUFMS-PIS03791 \\
\hline Prionobrama paraguayensis (Eigenmann 1914) & DZSJRP-12861 \\
\hline Astyanax abramis (Jenyns, 1842) & DZSJRP12873 \\
\hline Astyanax lacustris (Lütken 1875) (syn. Astyanax asuncionensis) & ZUFMS-PIS03848 \\
\hline Astyanax aff. fasciatus (Cuvier 1819) & NUP-17163 \\
\hline Astyanax lineatus (Perugia 1891) & NUP-17166 \\
\hline Astyanax marionae Eigenmann 1911 & NUP17197 \\
\hline Astyanax sp. 1 & NUP-17162 \\
\hline Astyanax sp. 2 & NUP-17178 \\
\hline Astyanax sp. 3 & NUP-17161 \\
\hline Hyphessobrycon cf. arianae Uj \& Géry 1989 & DZSJRP-12898 \\
\hline Hyphessobrycon eques (Steindachner 1882) & DZSJRP-12863 \\
\hline Hyphessobrycon elachys Weitzman 1984 & DZSJRP-12866 \\
\hline Hyphessobrycon luetkenii (Boulenger 1887) & DZSJRP-11969 \\
\hline Psellogrammus kennedyi (Eigenmann 1903) & ZUFMS-PIS01427 \\
\hline Brachychalcinus retrospina Boulenger 1892 & NUP-17213 \\
\hline
\end{tabular}

${ }^{1}$ Sabino \& Trajano (1997); ${ }^{2}$ Ribeiro et al. (2007); ${ }^{3}$ Zawadzki et al. (2014); ${ }^{4}$ Terra \& Sabino (2007); ${ }^{5}$ Willink et al. (2000); 6Rizzato, Costa, Trajano \& Bichuette (2011); ${ }^{7}$ Shibatta (2016). * Species occurrence information, but not sampled. 
Ferreira, F.S. et al.

Table 1: Continued...

\begin{tabular}{|c|c|}
\hline Taxon & Voucher \\
\hline Bryconops melanurus (Bloch 1794) & DZSJRP-12917 \\
\hline Hemigrammus lunatus Durbin 1918 & DZSJRP-12882 \\
\hline Hemigrammus tridens Eigenmann 1907 & DZSJRP-12885 \\
\hline Moenkhausia bonita Benine, Castro \& Sabino 2004 & NUP-17165 \\
\hline Moenkhausia forestii Benine, Mariguela \& Oliveira 2009 & ZUFMS-PIS04042 \\
\hline Moenkhausia intermedia Eigenmann 1908 & ZUFMS-PIS03832 \\
\hline Moenkhausia oligolepis (Günter 1864) & ZUFMS-PIS03863 \\
\hline Moenkhausia sanctafilomenae (Steindachner 1907) & ZUFMS-PIS00173 \\
\hline Jupiaba acanthogaster (Eigenmann 1911) & NUP-17168 \\
\hline Gymnocorymbus ternetzi (Boulenger 1895) & ZUFMS-PIS01449 \\
\hline Poptella paraguayensis (Eigenmann 1907) & ZUFMS-PIS01450 \\
\hline Bryconamericus exodon (Eigenmann 1907) & NUP-17196 \\
\hline Bryconamericus stramineus Eigenmann 1908 & NUP-17205 \\
\hline Creagrutus meridionalis Vari \& Harold 2001 & NUP-17195 \\
\hline Piabarchus analis (Eigenmann 1914) & NUP-17190 \\
\hline Piabarchus torrenticola Mahnert \& Géry 1985 & NUP-17214 \\
\hline Xenurobrycon macropus Myers \& Miranda Ribeiro 1945 & DZSJRP-12881 \\
\hline Oligosarcus perdido Ribeiro, Cavallaro \& Froehlich 2007 & ${ }^{2} \mathrm{LIRP}-5893$ \\
\hline Oligosarcus pintoi Campos 1945 & ZUFMS-PIS04038 \\
\hline Tetragonopterus argenteus (Cuvier 1816) & ZUFMS-PIS01541 \\
\hline \multicolumn{2}{|l|}{ Crenuchidae } \\
\hline Characidium borellii (Boulenger 1895) & ZUFMS-PIS02505 \\
\hline Characidium aff. fasciatum Reinhardt 1866 & DZSJRP-13743 \\
\hline Characidium laterale (Boulenger 1895) & DZSJRP-12855 \\
\hline Characidium aff. zebra Eigenmann 1909 & NUP-17172 \\
\hline Characidium cf. gomesi Travassos 1956 & DZSJRP-12876 \\
\hline Characidium sp. 1 & NUP-17559 \\
\hline Characidium sp. & NUP-17181 \\
\hline \multicolumn{2}{|l|}{ Curimatidae } \\
\hline Cyphocharax gillii (Eigenmann \& Kennedy 1903) & ZUFMS-PIS03850 \\
\hline Steindachnerina brevipinna (Eigenmann \& Eigenmann 1889) & ZUFMS-PIS03794 \\
\hline \multicolumn{2}{|l|}{ Erythrinidae } \\
\hline Hoplias sp. 1 & ZUFMS-PIS03877 \\
\hline Erythrinus erythrinus (Bloch \& Schneider 1801) & DZSJRP-12900 \\
\hline Hoplerythrinus unitaeniatus (Agassiz 1829) & DZSJRP-12902 \\
\hline \multicolumn{2}{|l|}{ Iguanodectidae } \\
\hline Piabucus melanostoma Holmberg 1891 & DZSJRP-000620 \\
\hline \multicolumn{2}{|l|}{ Lebiasinidae } \\
\hline Pyrrhulina australis Eigenmann \& Kennedy 1903 & DZSJRP-12864 \\
\hline \multicolumn{2}{|l|}{ Parodontidae } \\
\hline Apareiodon affinis (Steindachner 1879) & DZSJRP-12910 \\
\hline Parodon nasus Kner 1859 & ZUFMS-PIS04057 \\
\hline \multicolumn{2}{|l|}{ Prochilodontidae } \\
\hline Prochilodus lineatus (Valenciennes 1836) & LIRP-5849 \\
\hline \multicolumn{2}{|l|}{ Serrasalmidae } \\
\hline Metynnis maculatus (Kner 1858) & MZUEL-5235 \\
\hline Piaractus mesopotamicus (Holmberg 1887) & ${ }^{5}$ DZSJRP-007798 \\
\hline Serrasalmus maculatus Kner 1858 & ${ }^{5}$ MZUEL-14023 \\
\hline Serrasalmus marginatus Valenciennes 1837 & ZUFMS-PIS03836 \\
\hline \multicolumn{2}{|l|}{ GYMNOTIFORMES } \\
\hline \multicolumn{2}{|l|}{ Apteronotidae } \\
\hline Apteronotus albifrons (Linnaeus 1766) & DZSJRP-12407 \\
\hline \multicolumn{2}{|l|}{ Gymnotidae } \\
\hline Gymnotus inaequilabiatus (Valenciennes 1839) & DZSJRP-12856 \\
\hline Gymnotus sp. & ZUFMS-PIS04043 \\
\hline Hipopomidae & \\
\hline
\end{tabular}

${ }^{1}$ Sabino \& Trajano (1997); ${ }^{2}$ Ribeiro et al. (2007); ${ }^{3}$ Zawadzki et al. (2014); ${ }^{4}$ Terra \& Sabino (2007); ${ }^{5}$ Willink et al. (2000); 6Rizzato, Costa, Trajano \& Bichuette (2011); ${ }^{7}$ Shibatta (2016). * Species occurrence information, but not sampled. 
Table 1: Continued...

\begin{tabular}{|c|c|}
\hline Taxon & Voucher \\
\hline Brachyhypopomus sp. B & MZUSP-59444.0 \\
\hline Brachyhypopomus sp. C & ZUFMS-PIS3820 \\
\hline Brachyhypopomus sp. & DZSJRP-12862 \\
\hline \multicolumn{2}{|l|}{ Rhamphichthyidae } \\
\hline Gymnorhamphichtys britskii Carvalho, Ramos \& Albert, 2011 & ZUFMS-PIS01487 \\
\hline \multicolumn{2}{|l|}{ Sternopygidae } \\
\hline Eigenmannia trilineata López \& Castello 1966 & DZSJRP-12865 \\
\hline Sternopygus macrurus (Bloch \& Schneider 1801) & ZUFMS-PIS03397 \\
\hline \multicolumn{2}{|l|}{ SILURIFORMES } \\
\hline \multicolumn{2}{|l|}{ Auchenipteridae } \\
\hline Tatia neivai (Ihering 1930) & ZUFMS-PIS01419 \\
\hline \multicolumn{2}{|l|}{ Callichthyidae } \\
\hline Callichthys callichthys (Linnaeus 1758) & ZUFMS-PIS04046 \\
\hline Hoplosternum littorale (Hancock 1828) & ZUFMS-PIS01132 \\
\hline Lepthoplosternum pectorale (Boulenger 1895) & DZSJRP-12901 \\
\hline Corydoras aeneus (Gill 1858) & DZSJRP-12886 \\
\hline Corydoras areio Knaack 2000 & ZUFMS-PIS01105 \\
\hline Corydoras hastatus Eigenmann \& Eigenmann 1888 & DZSJRP-12904 \\
\hline Corydoras sp. & ZUFMS-PIS05366 \\
\hline \multicolumn{2}{|l|}{ Cetopsidae } \\
\hline Cetopsis gobioides (Kner 1858) & ZUFMS-PIS04039 \\
\hline \multicolumn{2}{|l|}{ Heptapteridae } \\
\hline Imparfinis mirini Haseman 1911 & ZUFMS-PIS02493 \\
\hline Imparfinis cf. schubarti (Gomes 1956) & DZSJRP-12911 \\
\hline Imparfinis stictonotus (Fowler 1840) & NUP-17194 \\
\hline Imparfinis sp. 1 & DZSJRP-12913 \\
\hline Imparfinis sp. 2 & DZSJRP-12887 \\
\hline Imparfinis sp. & NUP-17180 \\
\hline Pimelodella gracilis (Valenciennes in d'Orbigny 1835) & DZSJRP-12914 \\
\hline Pimelodella taenioptera Miranda-Ribeiro 1914 & ZUFMS-PIS01052 \\
\hline Phenacorhamdia hoehnei (Miranda-Ribeiro 1914) & DZSJRP-12410 \\
\hline Rhamdia quelen (Quoy \& Gaimard 1824) & ZUFMS-PIS01046 \\
\hline \multicolumn{2}{|l|}{ Loricariidae } \\
\hline Otocinclus bororo Schaefer 1997 & ${ }^{4} \mathrm{NUP}-13347$ \\
\hline Otocinclus vittatus Regan 1904 & DZSJRP-12874 \\
\hline Hypostomus basiliskoTencatt, Zawadzk i\& Froehlich 2014 & ZUFMS-PIS01294 \\
\hline Hypostomus boulengeri (Eigenmann \& Kennedy 1903) & DZSJRP-12908 \\
\hline Hypostomus cochliodon Kner 1854 & DZSJRP-12437 \\
\hline Hypostomus khimaeraTencatt, Zawadzki \& Froehlich 2014 & NUP-17207 \\
\hline Hypostomus aff. latirostris (Regan 1904) & NUP-17170 \\
\hline Hypostomus piratatu Weber 1986 & ZUFMS-PIS02539 \\
\hline Hypostomus perdido Zawadzki, Tencatt \& Froehlich 2014 & ${ }^{3}$ ZUFMS-PIS1468 \\
\hline Hypostomus sp. & NUP-17537 \\
\hline Ancistrus formoso Sabino \& Trajano 1997 & ${ }^{1}$ MZUSP-51836 \\
\hline Ancistrus sp. & NUP-17209 \\
\hline Farlowella cf. isbrueckeri Retzer \& Page 1997 & ZUFMS-PIS01291 \\
\hline Farlowella paraguayensis Retzer \& Page 1997 & NUP-17202 \\
\hline Loricaria cf. coximensis Rodriguez, Cavallaro \& Thomas 2012 & NUP-17204 \\
\hline Loricaria luciae Thomas, Rodriguez, Cavallaro, Froehlich \& Macedo Corrêa e Castro 2013 & NUP-17171 \\
\hline Loricaria sp. & DZSJRP-12920 \\
\hline Proloricaria prolixa Isbrücker \& Nijssen 1978 & MZUEL-10006 \\
\hline Pyxiloricaria menezesi Isbrücker \& Nijssen 1984 & NUP-17203 \\
\hline Rineloricaria cacerensis (Miranda-Ribeiro 1912) & ZUFMS-PIS01344 \\
\hline Rineloricaria lanceolata (Günther 1868) & NUP-17192 \\
\hline Rineloricaria parva (Boulenger 1895) & ZUFMS-PIS03839 \\
\hline Pimelodidae & \\
\hline
\end{tabular}

${ }^{1}$ Sabino \& Trajano (1997); ${ }^{2}$ Ribeiro et al. (2007); ${ }^{3}$ Zawadzki et al. (2014); ${ }^{4}$ Terra \& Sabino (2007); ${ }^{5}$ Willink et al. (2000); 6Rizzato, Costa, Trajano \& Bichuette (2011); ${ }^{7}$ Shibatta (2016). * Species occurrence information, but not sampled. 
Table 1: Continued...

\begin{tabular}{|c|c|}
\hline Taxon & Voucher \\
\hline Pseudoplatystoma corruscans (Spix \& Agassiz 1829) & MZUSP-51269.0 \\
\hline Pseudoplatystoma reticulatum Eigenmann \& Eigenmann 1889 & $* 5$ \\
\hline \multicolumn{2}{|l|}{ Pseudopimelodidae } \\
\hline Microglanis leniceae Shibatta 2016 & ZUFMS-PIS01657 ${ }^{7}$ \\
\hline Pseudopimelodus mangurus (Valenciennes 1835) & ZUFMS-PIS03397 \\
\hline Rhyacoglanis pulcher (Boulenger 1887) & DZSJRP-12141 \\
\hline \multicolumn{2}{|l|}{ Trichomycteridae } \\
\hline Paravandellia oxyptera Miranda-Ribeiro 1912 & DZSJRP-12877 \\
\hline Trichomycterus dali Rizzato, Costa, Trajano \& Bichuette 2011 & MZUSP $166630^{6}$ \\
\hline Trichomycterus sp. & NUP-9351 \\
\hline \multicolumn{2}{|l|}{ CYPRINODONTIFORMES } \\
\hline \multicolumn{2}{|l|}{ Poeciliidae } \\
\hline Poecillia reticulata Peters 1859 & ZUFMS-PIS03799 \\
\hline \multicolumn{2}{|l|}{ Cynolebiidae } \\
\hline Melanorivulus punctatus (Boulenger 1895) & DZSJRP-12905 \\
\hline \multicolumn{2}{|l|}{ SYNBRANCHIFORMES } \\
\hline \multicolumn{2}{|l|}{ Synbranchidae } \\
\hline Synbranchus marmoratus Bloch 1795 & ZUFMS-PIS03797 \\
\hline \multicolumn{2}{|l|}{ CICHLIFORMES } \\
\hline \multicolumn{2}{|l|}{ Cichlidae } \\
\hline Aequidens plagiozonatus Kullander 1984 & DZSJRP-12906 \\
\hline Bujurquina vittata (Heckel 1840) & ZUFMS-PIS03843 \\
\hline Cichlassoma dimerus (Heckel 1840) & NUP-17187 \\
\hline Crenicichla lepidota Heckel 1840 & DZSJRP-12889 \\
\hline Crenicichla vittata Heckel 1840 & ${ }^{5}$ ZUFMS-PIS01307 \\
\hline Crenicichla sp. & ZUFMS-PIS03800 \\
\hline
\end{tabular}

${ }^{1}$ Sabino \& Trajano (1997); ${ }^{2}$ Ribeiro et al. (2007); ${ }^{3}$ Zawadzki et al. (2014); ${ }^{4}$ Terra \& Sabino (2007); ${ }^{5}$ Willink et al. (2000); 6Rizzato, Costa, Trajano \& Bichuette (2011); ${ }^{7}$ Shibatta (2016). * Species occurrence information, but not sampled.
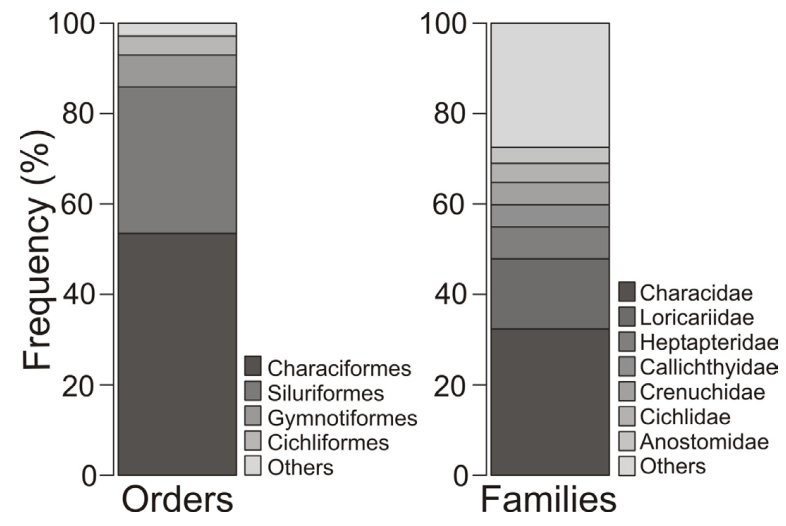

Figure 2: Frequency of orders and families from to primary and secondary data from the Miranda River Basin of the Upper Paraguay River, Mato Grosso do Sul, Brazil.

of Near-threatened Species. Furthermore Characidium aff. fasciatum Reinhardt 1866, Farlowella cf. isbrueckeri Retzer \& Page 1997 are listed as data deficient. The cave fishes Ancistrus formoso Sabino \& Trajano 1997 and Trichomycterus dali Rizzato, Costa, Trajano \& Bichuette 2011 are listed as vulnerable. We encountered six possible new species, including Astyanax sp. 1, Astyanax sp. 2, Astyanax sp. 3, Characidium sp. 1, Imparfinis sp. 1, Imparfinis sp. 2. Six species from our data sets were reported as only being found in the Paraná River basin, such as Serrapinnus notomelas (Eigenmann 1915), Astyanax aff. fasciatus (Cuvier 1819), Imparfinis mirini Haseman 1911, Imparfinis cf. schubarti

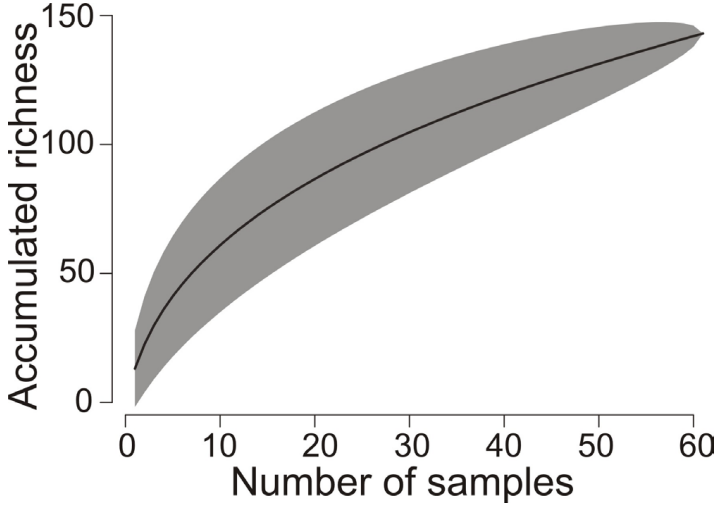

Figure 3: Species accumulated curve generated from to primary and secondary data from the Miranda River Basin of the Upper Paraguay River, Mato Grosso do Sul, Brazil.

(Gomes 1956), Proloricaria prolixa Isbrücker \& Nijssen 1978 and Hoplias sp. 1 from our primary data set, and Oligosarcus pintoi Campos 1945, Rineloricaria lanceolata (Günter 1868) from our secondary data set.

\section{Discussion}

Studies of fishes from the Paraguay River basin usually concentrate on the main rivers of the Pantanal region (Britski et al. 2007, Terra \& Sabino 2007, Polaz et al. 2014), due mainly to the easy access into these areas, when compared to the headwaters, this makes the knowledge of the 
fauna of streams almost non-existent (Krinski et al. 2015). However, to understand the environmental structural dynamics, the accomplishment of ichthyofauna inventories and systematic studies are extremely important. Besides, they constitute the basis for better understanding the dynamics of species distribution (Vilar et al. 2011), providing data on the species composition, management and conservation (Pains-Silva et al. 2014).

Our results showed predominance of Characiformes and Siluriformes, and the families Characidae and Loricariidae. Other studies conducted in streams of the Paraguay River Basin reported similar results (Oliveira et al. 2015, Vizzotto \& Castro 2015), which reflect typical species patterns for Neotropical icthyofauna (Reis et al. 2016).

The combination of primary and secondary data for the Miranda River Basin produced a data set from a greater number of sample sites, over longer time periods, and at larger spatial scales, and the end product is a more complete assessment of regional fish fauna. Other studies of fish fauna in the Paraguay River Basin at smaller spatial scales report fewer numbers of species, including studies from streams of the headwaters of the Rio Vermelho (Oliveira et al. 2015, Vizzotto \& Castro 2015) and Rio Sepotuba (Krinski et al. 2015) in northern Pantanal. In other words, by increasing spatial scale and the number of sample sites, we also increase the probability of greater among-site variation in species numbers by virtue of including a greater diversity of mesohabitats with different substrate types and riparian vegetation. These differences in habitat structural complexity (Teresa et al. 2010, Teresa Romero \& 2010, Krinski et al. 2015, Vizzotto \& Castro 2015) result in a gradient of heterogeneity that, through niche differentiation, favors a greater number of species.

Some species in the current study should be highlighted due to conservation status. Serrasalmidae and Pimelodidae, Piaractus mesopotamicus and Pseudoplatystoma corruscans are on the National List of Near-threatened Species (NT) in Brazil (data published by ICMBio 2014), despite this, these species have broad distribution in the Paraguay River Basin, in addition, they are important species for artisanal and sports fishing in the region, being $P$. corruscans represent approximately $32 \%$ and $P$. mesopotamicus $17.3 \%$ of artisanal fisheries in 2015 (Catella et al. 2016). So, this results suggest a need of effort to better definition of conservation status at upper Paraguay basin scale.

Among the species sampled were Characidium aff. fasciatum and Farlowella cf. isbrueckeri from the families Crenuchidae and Loricariidae, respectively, both of which are listed at Brazilian law as Data Deficient (DD) (data published by ICMBio 2014) due to low numbers of recorded occurrences or absence in study samples. Ancistrus formoso and Trichomycterus dali are the only encountered species listed as vulnerable, likely a result of its limited distribution due to restriction to cave habitats in the karst environments of the Serra da Bodoquena (Cordeiro et al. 2014); the restricted range and the frequent change of the landscape to harbor crop fields in the region represent a great menace to these species. The Miranda River Basin seems to offers sufficient resources to maintain numerous species, in particular species of concern with respect to conservation. The collective data thus suggest that preservation of this basin ecosystem is critical for perpetuation of natural resources and biodiversity.

There are roughly 270 described species in the Pantanal floodplain (Britski et al. 2007), and our list presents 39 additional species not included in that estimate. Several species from our data sets were reported as only being found in the Paraná River basin, this suggests dispersion during the formation of the Pantanal, and serves as evidence of connectivity between the headwaters of the Paraná and Paraguay River Basins. This finding illustrates the importance of expanding sampling effort for interface regions of the basins, and reviewing the material deposited in biological collections.
In addition to future studies of species richness in the Paraguay River Basin, more detailed information on species distributions is needed to better gauge conservation status of fish in the area. There have been a limited number of studies in the area, particularly in headwater streams and their tributaries (Oyakawa \& Menezes 2011, Krinski et al. 2015). Many species of headwater streams are potentially geographically isolated (Lowe-McConnell 1999) that are specific to these areas (Krinski et al. 2015), whereby headwaters may host very different fauna than exists in major rivers (Pedroza et al. 2012, Volcan et al. 2012). Based on previous experience and distribution maps from SpeciesLink, we can point out in this whor Astyanax lineatus, A. marionae, Creagrutus meridionalis, Oligosarcus perdido, Hypostomus basilisko, H. perdido and the cave fish Ancistrus formoso and Trichomycterus dali as exclusive species that inhabit headwaters. This understanding of fish fauna in headwater streams and associated tributaries is essential for maintaining fish biodiversity in the Paraguay River Basin. Inventory studies in the basin would also be useful for evaluation of potential environmental impacts on ichthyofauna, as these environments are subject to anthropogenic modification (e.g., due to the construction of hydroelectric dams, which has increased in last years). Such anthropogenic impacts have potential to modify species distribution (Mariano et al. 2012), by limiting species dispersion and altering resource availability in the entire plateau region of the Upper Paraguay Basin. In the Miranda River Basin, at least eleven small hydroelectric projects have recently approved for construction, two in Miranda sub-basin and nine in Aquidauana sub-basin. More attention should be given to the actual and potential negative impacts of these and other anthropogenic activities upon fish diversity and distributions in the basin area.

\section{Acknowledgments}

We thank the Biota-MS program in association with the Superintendência de Ciência e Tecnologia de MatoGrosso do Sul (SUCITEC), CAPES, CNPq and FUNDECT for financial and logistical support. We are grateful to Walmir B. F. Mundim Jr., Wagner Vicentin, Marcelo S. Maldonado and Gabriel Nakamura for help with field work.

\section{Author Contributions}

Fabiane S. Ferreira, Gabriela S. V. Duarte, Francisco Severo Neto, Otávio Froehlich and Yzel R. Súarez contributed to the field work and in secondary data collection. FSF and YRS also realized a statistical analysis and all authors contributed to manuscript preparation and critical revision.

\section{Conflicts of Interest}

The authors declare that they have no conflict of interest related to the publication of this manuscript.

\section{References}

ALHO, C.J.R. \& SABINO, J. 2012. Seasonal Pantanal flood pulse: implications for biodiversity conservation -A review. Oecol. Aust. 16(4): 958-978. doi: http:// dx.doi.org/10.4257/oeco.2012.1604.17.

BAGINSKI, L.J., FLORENTINO, A.C., FERNANDES, I.M., PENHA, J.M.P. \& MATEUS, L.A.F. 2007 The spatial and temporal dimension of fish diversity of the vegetated littoral zone of marginal lagoons of the Cuiabá river floodplain, Pantanal, Brazil. Biota Neotrop 7(3): 233-238. http://www.biotaneotropica.org. br/v7n3/pt/abstract?article+bn04007032007 (Accessed 24 Jun 2016).

BOTINI, A.F., BARROS, C.A., SOUZA, T.H., BOTINI, N., MOURA, N.A. 2015. Diversidade de peixes no Rio Mutum e baía marginal no Pantanal-Matogrossense através da coleta ativa. Enciclop Biosf 11(21): 2183-2197. 
BRITSKI, H.A., SILIMON, K.Z.S. \& LOPES, B.S. 2007. Peixes do Pantanal: manual de identificação. 2 ed. Embrapa, Brasília, 227p.

CASATTI, L., ROMERO, R.M., TERESA, F.B., SABINO, J. \& LANGEANI, F. 2010. Fish community structure along a conservation gradient in Bodoquena Plateau streams, central West of Brazil. Acta Limnol Brasil 22(1): 50-59. doi: http://dx.doi.org/10.4322/actalb.02201007.

CASTRO, R.J. \& VIZZOTTO, P.C. 2013. Fishes of the Vermelho River, São Lourenço River basin, MatoGrosso state, Brazil. Check List 9(1): 001-003.

CATELlA, A.C., CAMPOS, F.L.R., ALBUQUERQUE, S.P. 2016. Sistema de controle de pesca de Mato Grosso do Sul SCPESCA/MS 22- 2015. Corumbá: Boletim de pesquisa e desenvolvimento - Embrapa Pantanal. p. 55.

CATELLA, A.C. \& PETRERE-JR, M. 1996. Feeding patterns in a fish community of Baia da Onça, a foodplain lake of the Aquidauana River, Pantanal, Brazil. Fisheries Manag Ecol 3: 229-237.

CORDEIRO, L.M.; BORGHEZAN, R. \& TRAJANO, E. 2014. Subterranean biodiversity in the Serra da Bodoquena karst area, Paraguay river basin, Mato Grosso do Sul, Southwestern Brazil. Biota Neotropica14: 1-28. doi: http:/ dx.doi.org/10.1590/1676-06032014011414.

ESCHMEYER, W.N., FRICKE, R. \& VAN DER LAAN, R. (eds.). 2017. Catalog of Fishes: Genera, Species, References.http://researcharchive.calacademy. org/research/ichthyology/catalog/fishcatmain.asp (Accessed 20 May 2017).

JESUS, F. 2003. Classificação dos ecossistemas aquáticos do Pantanal e da bacia do Alto Paraguai. Brasília: The Nature Conservancy. p. 136.

JUNK, W.J., CUNHA, C.N., WANTZEN, K.M., PETERMANN, P., STRÜSSMANN, C., MARQUES, M.I. \& ADIS, J. 2006. Biodiversity and its conservation in the Pantanal of Mato Grosso, Brazil. Aquat Sci 68: 278-309. doi: http://dx.doi org/10.1007/s00027-006-0851-4.

KRINSKI, D., CARMO, E.J. \& MIYAZAWA, C.S. 2015. Ichthyofauna of headwaters from Sepotuba River Basin, Upper Paraguay River Basin, Tangará da Serra, Mato Grosso state, Brazil. Panamjas 10(4): 283-292.

MARIANO, J.R., MAKRAKIS, M.C., KASHIWAQUI, E.A.L., CELESTINO, E.F. \& MAKRAKIS, S. 2012. Longitudinal habitat disruption in Neotropical streams: fish assemblages under the influence of culverts. Neotrop Ichthyol 10(4):771-784. doi: http://dx.doi.org/10.1590/S1679-62252012000400010.

OKSANEN, J., BLANCHET, F.G., KINDT, R., LEGENDRE, P., O'HARA, R.B., SIMPSON, G.L., STEVENS, M.H.H. \& WAGNER, H. 2011. Vegan: community ecology package. Version 1.17-11. Available from: http://vegan.rforge.r-project.org/

OLIVEIRA, V.A., MATEUS, L.A., LOVERDE-OLIVEIRA, S. \& PIETRO-SOUZA, W. 2015. Fish from urban tributaries to the Vermelho River, upper Paraguay River Basin, Mato Grosso, Brazil. Check List 11(1): 1-6. doi: http://dx.doi. org/10.15560/11.1.1516.

OYAKAWA, O.T. \& MENEZES, N.A. 2011. Checklist of fresh water fishes from São Paulo State, Brazil. Biota Neotrop 11(1a): 19-32. doi: http://dx.doi.org/10.1590/ S1676-06032011000500002.

PACHECO, E.B. \& SILVA, C.J. 2009. Fish associated with aquatic macrophytes in the Chacororé-Sinhá Mariana lake system and Mutum River, Pantanal of Mato Grosso Brazil. Braz J Biol 69(1): 101-108. doi: http://dx.doi.org/10.1590/ S1519-69842009000100012.

PAINS-SILVA, H., SOUZA, F. \& CORREAA, F. 2014. Icththyofauna of the Bento Gomes River in the Pantanal Matogrossense, Brazil. Panamjas 9(1): 48-53.

PAINS-SILVA, H., PETRY, A.C. \& DA SILVA, C.J. 2010. Fish communities of the Pantanal wetland in Brazil: evaluating the effects of the Upper Paraguay River flood pulse on baía Caiçara fish fauna. Aquat Ecol 44: 275-288. doi: http:// dx.doi.org/10.1007/s10452-009-9289-9.

PEDROZA, W.S., RIBEIRO, F.R.V., TEIXEIRA, T.F., OHARA, W.M. \& PY-DANIEL, L.H.R. 2012. Icththyofauna survey of stretches of the Guariba and Roosevelt Rivers, in Guariba State Park and Guariba Extractive Reserve, Madeira River basin, Amazonas, Brazil. Check List 8(1): 8-15.

PLANO DE RECURSOS HÍDRICOS DA BACIA HIDROGRÁFICA DO RIO MIRANDA. 2014. http://www.imasul.ms.gov.br/wp-content/uploads/ sites/74/2015/06/Diagn\%C3\%B3stico-Final-Plano-de-Bacia-Miranda.pdf (Acessed 20 May 2017).
POLAZ, C.N.M., MELO, B.F., BRITZKE, R., RESENDE, E.K., MACHDO, F.A., LIMA, J.A.F. \& PETRERE jr, M. 2014. Fishes from the Parque Nacional do Pantanal Matogrossense, upper Paraguai River basin, Brazil. Check List 10(1): 122-130. doi: http://dx.doi.org/10.15560/10.1.122.

R DEVELOPMENT CORE TEAM. 2013. R: A language and environment for statistical computing. R Foundation for Statistical Computing, Vienna, Austria http://www.R-project.org/.

REIS, R.E., ALBERT, J.S., DI DARIO, F., MINCARONE, M.M., PETRY, P. \& ROCHA, L.A. 2016. Fish biodiversity and conservation in South America. J. Fish Biol.89(1):12-47. doi: http://dx.doi.org/10.1111/jfb.13016

RIBEIRO, A.C., CAVALLARO, M.R. \& FROEHLICH, O. 2007. Oligosarcus perdido (Characiformes, Characidae), a new species of fresh water fish from Serra da Bodoquena, upper Rio Paraguai basin, Brazil. Zootaxa 1560: 43-53.

SABINO, J. \& TRAJANO, E. 1997. A new species of blind armoured catfish, genus Ancistrus, from caves of Bodoquena region, Mato Grosso do Sul, southwestern Brazil (Siluriformes, Loricariidae, Ancistrinae). Revue Fr Aquariol 23: 73-78.

SEVERO-NETO, F., TENCATT, L.F.C., COSTA-PEREIRA, R. \& TAVARES L.E.R. 2015. Fishes from Baía da Medalha, southern Pantanal, Brazil: A 20 years review. Biota Neotrop 15(2): 1-9. doi: http://dx.doi.org/10.1590/167606032015011614

SILVA, J.S.V. \& ABDON, M.M. 1998. Delimitação do Pantanal Brasileiro e suas sub-regiões. Pesq. Agropec. Bras. 33: 1703-1711.

SHIBATTA, O.A. 2016. A new species of bumblebee catfish of the genus Microglanis (Siluriformes: Pseudopimelodidae) from the upper rio Paraguay basin, Brazil Neotrop Ichthyol 14(3): e160031. http://dx.doi.org/10.1590/1982-0224-20160031.

SMITH, E.P. \& VAN BELLE, G. 1984. Nonparametric estimation of species richness. Biometrics 40(1):119-129. doi: http://dx.doi.org/10.2307/2530750.

SÚAREZ, Y.R., FERREIRA, F.S. \& TONDATO, K.K. 2013. Assemblage of fish species associated with aquatic macrophytes in Porto Murtinho Pantanal, Mato Grosso do Sul, Brazil. Biota Neotrop 13(2): 182-189. http://www.biotaneotropica. org.br/v13n2/en/abstract?inventory+bn02313022013 (Accessed 24 Jun 2016)

SÚAREZ, Y.R., VALÉRIO, S.B., TONDATO, K.K., FLORENTINO, A.C., FELIPE, T.R.A., XIMENES, L.Q.L. \& LOURENÇO, L.S. 2007. Fish species diversity in headwaters streams of Paraguay and Paraná basins. Braz Arch Biol Techn 50(6): 1033-1042. doi: http://dx.doi.org/10.1590-89132007000700014.

TERESA, F.B. \& ROMERO, R.M. 2010. Influence of the riparian zone phytophysiognomies on the longitudinal distribution of fishes: evidence from a Brazilian savanna stream. Neotrop Ichthyol 8(1): 163-170. doi: http://dx.doi org/10.1590/S1679-62252010000100019.

TERESA, F.B., ROMERO, R.M. \& LANGEANI, F. 2010. Pisces, Aquidauana and Miranda drainages, upper Paraguay River basin, Mato Grosso do Sul, Brazil Check List 6(4): 596-601.

TERRA, L.C.C. \& SABINO, J. 2007. Composição da ictiofauna de dois riachos, com diferentes graus de conservação, na bacia do Rio Formoso, município de Bonito, Mato Grosso do Sul, Brasil. Redalyc 11(1): 49-58. http://www.redalyc. org/articulo.oa?id=26012838005 (Accessed 24 Jun 2016).

TONDATO, K.K., FANTIN-CRUZ, I., PEDROLLO, O.C., SÚAREZ, Y.R. 2013. Spatial distribution of fish assemblages along environmental gradients in the temporary ponds of Northern Pantanal, Brazil. J Limnol 71(1): 95-102. doi: http://dx.doi.org/10.4081/jlimnol.2013.e8.

VILAR, C.C., SPACH, H.L. \& SOUZA-CONCEIÇÃO, J.M. 2011. Fish assemblage in shallow areas of Baía da Babitonga, southern Brazil: structure, spatial and temporal patterns. Panamjas 6(4): 303-319.

VIZZOTO, P.C. \& CASTRO, R.J. 2015. Ichthyofauna of tributaries of the Vermelho River, upper Paraguay River basin, region of Rondonópolis, state of Mato Grosso, Brazil. Check List 11(5): 1-5. doi: http://dx.doi.org/10.15560/11.5.1750.

VOLCAN, M.V., LANÉS, L.E.K., GONÇALVES, A.C., FONSECA, A.P. \& CIRNE, M.P. 2012. The fish fauna of the Corrientes stream basin, Patos lagoon system, state of Rio Grande do Sul, southern Brazil. Check List 8(1): 77-82. doi: http:// dx.doi.org/10.15560/8.1.077.

WILLINK, P.W., FROEHLICH, O., MACHADO-ALISSON, A., MENEZES, N., OYAKAWA, O., CATELLA, A., CHERNOFF, B., LIMA, F.C.T., TOLEDOPIZA, M., HORTEGA, H., ZANATA, A.M. \& BARRIGA, R. 2000. Diversidade, distribuição e habitats críticos dos peixes dos rios: Negro, Negrinho, Taboco, 
Taquari e Miranda, e sua importância para conservação e desenvolvimento sustentável do Pantanal, Mato Grosso do Sul, Brasil. Pp. 63-81. In: WILLINK, P. W., CHERNOFF, B., ALONSO, L., MONTAMBAULT, J.R. \& LOURIVAL, R. (eds). RAP Bulletin of Biological Assesment, 18: Uma Avaliação Biológica dos Ecossistemas Aquáticos do Pantanal, Mato Grosso do Sul, Brasil. Conservation International, Washington.

ZAWADZKI, C.H., L.F.C. TENCATT, and O. FROEHLICH. 2014. A new unicuspidtoothed species of Hypostomus Lacépède, 1803 (Siluriformes: Loricariidae) from the rio Paraguai basin. Neotrop Ichthyol 12(1): 97-104. doi: http://dx.doi. org/10.1590/S1679-62252014000100010.

Received: $15 / 03 / 2017$

Revised: 20/07/2017

Accepted: 21/07/2017

Published online: 14/08/2017 